PROCEEDINGS OF THE

AMERICAN MATHEMATICAL SOCIETY

Volume 140, Number 5, May 2012, Pages 1681-1696

S 0002-9939(2011)11011-6

Article electronically published on September 2, 2011

\title{
STATIONARY SOLUTIONS AND SPREADING SPEEDS OF NONLOCAL MONOSTABLE EQUATIONS IN SPACE PERIODIC HABITATS
}

\author{
WENXIAN SHEN AND AIJUN ZHANG
}

(Communicated by Yingfei Yi)

\begin{abstract}
This paper deals with positive stationary solutions and spreading speeds of monostable equations with nonlocal dispersal in spatially periodic habitats. The existence and uniqueness of positive stationary solutions and the existence and characterization of spreading speeds of such equations with symmetric convolution kernels are established in the authors' earlier work for the following cases: the nonlocal dispersal is nearly local; the periodic habitat is nearly globally homogeneous or it is nearly homogeneous in a region where it is most conducive to population growth. The above conditions guarantee the existence of principal eigenvalues of nonlocal dispersal operators associated to linearized equations at the trivial solution. In general, a nonlocal dispersal operator may not have a principal eigenvalue. In this paper, we extend our earlier results to general spatially periodic nonlocal monostable equations. As a consequence, it is seen that the spatial spreading feature is generic for monostable equations with nonlocal dispersal.
\end{abstract}

\section{INTRODUCTION}

The current paper is an extension of the work [41] on spatial spreading dynamics of the following monostable equation with nonlocal dispersal:

$$
\frac{\partial u}{\partial t}=\int_{\mathbb{R}^{N}} k(y-x) u(t, y) d y-u(t, x)+u(t, x) f(x, u(t, x)), \quad x \in \mathbb{R}^{N},
$$

where $k(\cdot)$ is a $C^{1}$ convolution kernel supported on a ball centered at 0 (i.e. $k(z)>0$ if $\|z\|<\delta$ and $k(z)=0$ if $\|z\| \geq \delta$ for some $\delta>0$, where $\|\cdot\|$ denotes the norm in $\left.\mathbb{R}^{N}\right)$, and $\int_{\mathbb{R}^{N}} k(z) d z=1$. The function $f(x, u)$ is $C^{1}$ in $(x, u) \in \mathbb{R}^{N} \times \mathbb{R}$, periodic in $x_{i}$ with period $p_{i}\left(p_{i}>0, i=1,2, \cdots, N\right)$ (i.e. $f\left(\cdot+p_{i} e_{i}, \cdot\right)=f(\cdot, \cdot)$, $e_{i}=\left(\delta_{i 1}, \delta_{i 2}, \cdots, \delta_{i N}\right), \delta_{i j}=1$ if $i=j$ and 0 if $\left.i \neq j, i, j=1,2, \cdots, N\right)$, and satisfies proper monostablility assumptions. More precisely, let

$$
X_{p}=\left\{u \in C\left(\mathbb{R}^{N}, \mathbb{R}\right) \mid u\left(\cdot+p_{i} e_{i}\right)=u(\cdot), \quad i=1, \cdots, N\right\}
$$

with norm $\|u\|_{X_{p}}=\sup _{x \in \mathbb{R}^{N}}|u(x)|$, and

$$
X_{p}^{+}=\left\{u \in X_{p} \mid u(x) \geq 0 \quad \forall x \in \mathbb{R}^{N}\right\} .
$$

Received by the editors September 17, 2010 and, in revised form, January 17, 2011. 2010 Mathematics Subject Classification. Primary 45C05, 45G10, 45M20, 47G10, 92D25.

Key words and phrases. Monostable equation, nonlocal dispersal, random dispersal, periodic habitat, spreading speed, principal eigenvalue, principal eigenfunction, variational principle.

This work was partially supported by NSF grant DMS-0907752.

(C)2011 American Mathematical Society 1681

Reverts to public domain 28 years from publication 
Let $I$ be the identity map on $X_{p}$, and $K, a_{0}(\cdot) I: X_{p} \rightarrow X_{p}$ be defined by

$$
\begin{gathered}
(K u)(x)=\int_{\mathbb{R}^{N}} k(y-x) u(y) d y, \\
\left(a_{0}(\cdot) I u\right)(x)=a_{0}(x) u(x),
\end{gathered}
$$

where $a_{0}(x)=f(x, 0)$. The monostablility assumptions are then stated as follows:

(H1) $\frac{\partial f(x, u)}{\partial u}<0$ for $x \in \mathbb{R}^{N}$ and $u \in \mathbb{R}$ and $f(x, u)<0$ for $x \in \mathbb{R}^{N}$ and $u \gg 1$.

(H2) $u \equiv 0$ is linearly unstable in $X_{p}$; that is, $\lambda_{0}:=\sup \left\{\operatorname{Re} \lambda \mid \lambda \in \sigma\left(K-I+a_{0}(\cdot) I\right)\right\}$ is positive, where $\sigma\left(K-I+a_{0}(\cdot) I\right)$ is the spectrum of the operator $K-I+a_{0}(\cdot) I$ on $X_{p}$.

It is proved in [41] that if $k(\cdot)$ is symmetric (i.e. $k(-x)=k(x)$ for $x \in \mathbb{R}^{N}$ ) and $\lambda_{0}$ in $(\mathrm{H} 2)$ is an isolated algebraically simple eigenvalue of $K-I+a_{0}(\cdot) I$ with an eigenfunction in $X_{p}^{+}$, then (H1) and (H2) imply that (1.1) has exactly two equilibrium solutions in $X_{p}^{+}, u=0$ and $u=u^{+}$, and $u=0$ is linearly unstable and $u=u^{+}$is asymptotically stable in $X_{p}$ (see [41, Proposition 2.4]). These results will be extended to the general case in this paper, i.e., the case that only (H1) and (H2) are satisfied, which reflects the monostable feature of the assumptions (H1) and (H2).

Equation (1.1) is a nonlocal dispersal counterpart of the following local or random dispersal equation

$$
\frac{\partial u}{\partial t}=\Delta u+u f(x, u), \quad x \in \mathbb{R}^{N} .
$$

Random dispersal is essentially a local behavior which describes the movement of species between adjacent spatial locations and has been widely used to model the population spreading dynamics of species (see [1, 3], 4, [5, 13, 14, 27, 32, 44, [45, and the references therein). In contrast, nonlocal dispersal characterizes the movements and interactions of species between nonadjacent spatial locations. As the movements and interactions of many species in biology and ecology can occur between nonadjacent spatial locations, nonlocal dispersal has also been used to model the population spreading dynamics of species by many people (see [2, 7], [8], [9, [1], 12, [16, 22, 23], 26], and the references therein).

One of the central problems for (1.1) and (1.6) is to understand how fast the population spreads as time evolves. For example, letting $\xi \in S^{N-1}:=\left\{\xi \in \mathbb{R}^{N} \mid\|\xi\|=\right.$ $1\}$ and a given initial population $u_{0}$ satisfy for some $\delta_{0}>0$ that $u_{0}(x) \geq \delta_{0}$ for $x \in \mathbb{R}^{N}$ with $x \cdot \xi \ll-1$ and $u_{0}(x)=0$ for $x \in \mathbb{R}^{N}$ with $x \cdot \xi \gg 1(x \cdot \xi$ is the inner product of $x$ and $\xi$ ), how fast does the population invade into the region with no population initially?

The spatial spreading dynamics of (1.6) has been extensively studied since the pioneering works of Fisher [14 and Kolmogorov, Petrowsky, Piscunov [27] on the following special case of (1.6):

$$
\frac{\partial u}{\partial t}=\frac{\partial^{2} u}{\partial x^{2}}+u(1-u), \quad x \in \mathbb{R}
$$

which models the evolutionary takeover of a habitat by a fitter genotype. See, for example, [1, [3, [4, [5, [13, [17, [20], 21, 25], 28], 29], 30, 32, 34, 35, [38, 39], 43], 44], 45], and the references therein for the study of the spatial spreading dynamics of (1.6). It is well known that if (H1) holds and $u \equiv 0$ is 
a linearly unstable solution of (1.6), then (1.6) has a unique positive equilibrium $u^{+}(\cdot) \in X_{p}^{+}$which is asymptotically stable with respect to perturbations in $X_{p}$ and for every $\xi \in S^{N-1}$, there is a $c^{*}(\xi) \in \mathbb{R}$ such that for every $c \geq c^{*}(\xi)$, there is a traveling wave solution connecting $u^{+}$and $u^{-} \equiv 0$ and propagating in the direction of $\xi$ with speed $c$, and there is no such traveling wave solution of slower speed in the direction of $\xi$. Moreover, the minimal wave speed $c^{*}(\xi)$ has some important spreading properties (hence is also called the spreading speed) and has the following variational characterization. Let $\lambda(\xi, \mu)$ be the eigenvalue of

$$
\left\{\begin{array}{l}
\Delta u-2 \mu \sum_{i=1}^{N} \xi_{i} \frac{\partial u}{\partial x_{i}}+\left(a_{0}(x)+\mu^{2}\right) u=\lambda u, \quad x \in \mathbb{R}^{N}, \\
u\left(x+p_{i} e_{i}\right)=u(x), \quad i=1,2, \cdots, N, \quad x \in \mathbb{R}^{N}
\end{array}\right.
$$

with largest real part, where $a_{0}(x)=f(x, 0)$. (It is well known that $\lambda(\xi, \mu)$ is real and algebraically simple. $\lambda(\xi, \mu)$ is called the principal eigenvalue of (1.8) in the literature.) Then

$$
c^{*}(\xi)=\inf _{\mu>0} \frac{\lambda(\xi, \mu)}{\mu} .
$$

(See [3, 44, 5], 28, 34, 35], 45] and the references therein for the abovementioned properties.)

Recently, the nonlocal dispersal equation of the form (1.1) has also been studied by many authors. See, for example, [2, [7, 8], 10, [12, [15, [16, 19, [22, 23, 24, 26, 40], 41, for the study of spectral theory for nonlocal dispersal operators and the existence, uniqueness, and stability of nontrivial positive stationary solutions. See, for example, 9], 11, 44, 44] for the study of the existence of spreading speeds and traveling wave solutions connecting the trivial solution $u=0$ and a nontrivial positive stationary solution for some special cases of (1.1). See also [31, 33], 36] and the references therein for the study of entire solutions and traveling wave solutions of certain (delayed) nonlocal monostable systems.

In the very recent paper [41], the authors of the current paper explored the spatial spreading dynamics of (1.6) in the case that $k(\cdot)$ is symmetric and the nonlocal counterpart of the eigenvalue problem (1.8) possesses a principal eigenvalue. In such a case, the existing results on spreading speed of (1.6) have been well extended to (1.1). Note that a nonlocal dispersal operator may not have a principal eigenvalue (see an example in [41), which reveals some essential difference between nonlocal dispersal operators and random dispersal operators.

To be more precise, consider the following eigenvalue problem, which is a nonlocal counterpart of (1.8):

$$
\left(K_{\xi, \mu}-I+a(\cdot) I\right) v=\lambda v, \quad v \in X_{p},
$$

where $\xi \in S^{N-1}, \mu \in \mathbb{R}, a(x)$ is a smooth function periodic in $x_{i}$ with period $p_{i}>0$ (i.e. $\left.a\left(x+p_{i} e_{i}\right)=a(x)\right)$ for $i=1,2, \cdots, N$. The operator $a(\cdot) I$ has the same meaning as in (1.5) with $a_{0}(\cdot)$ being replaced by $a(\cdot)$, and $K_{\xi, \mu}: X_{p} \rightarrow X_{p}$ is defined by

$$
\left(K_{\xi, \mu} v\right)(x)=\int_{\mathbb{R}^{N}} e^{-\mu(y-x) \cdot \xi} k(y-x) v(y) d y .
$$

We point out the following relation between (1.1) and (1.10): if $u(t, x)=$ $e^{-\mu\left(x \cdot \xi-\frac{\lambda}{\mu} t\right)} \phi(x)$ with $\phi \in X_{p} \backslash\{0\}$ is a solution of the linearization of (1.1) at 
$u=0$, that is,

$$
\frac{\partial u}{\partial t}=\int_{\mathbb{R}^{N}} k(y-x) u(t, y) d y-u(t, x)+a_{0}(x) u(t, x), \quad x \in \mathbb{R}^{N},
$$

where $a_{0}(x)=f(x, 0)$, then $\lambda$ is an eigenvalue of (1.10) with $a(\cdot)=a_{0}(\cdot)$ or $K_{\xi, \mu}-$ $I+a_{0}(\cdot) I$ and $v=\phi(x)$ is a corresponding eigenfunction.

Definition 1.1. Let $\sigma\left(K_{\xi, \mu}-I+a(\cdot) I\right)$ be the spectrum of $K_{\xi, \mu}-I+a(\cdot) I$ on $X_{p}$.

(1) $\lambda_{0}(\xi, \mu, a):=\sup \left\{\operatorname{Re} \lambda \mid \lambda \in \sigma\left(K_{\xi, \mu}-I+a(\cdot) I\right)\right\}$ is called the principal spectrum point of $K_{\xi, \mu}-I+a(\cdot) I$.

(2) A number $\lambda(\xi, \mu, a) \in \mathbb{R}$ is called the principal eigenvalue of (1.10) or $K_{\xi, \mu}-$ $I+a(\cdot) I$ if it is an algebraically simple eigenvalue of $K_{\xi, \mu}-I+a(\cdot) I$ with an eigenfunction $v \in X_{p}^{+}$, and for every $\lambda \in \sigma\left(K_{\xi, \mu}-I+a(\cdot) I\right) \backslash\{\lambda(\xi, \mu, a)\}$, $\operatorname{Re} \lambda<\lambda(\xi, \mu, a)$.

Observe that if the principal eigenvalue $\lambda(\xi, \mu, a)$ of $K_{\xi, \mu}-I+a(\cdot) I$ exists, then $\lambda(\xi, \mu, a)=\lambda_{0}(\xi, \mu, a)$. If $\mu=0$, (1.10) is independent of $\xi$, and hence we put

$$
\lambda_{0}(a):=\lambda_{0}(\xi, 0, a) \quad \forall \xi \in S^{N-1} .
$$

In terms of the principal eigenvalue, we make the following assumption:

(H3) The principal eigenvalue $\lambda\left(\xi, \mu, a_{0}\right)$ of (1.10) with $a(\cdot)=a_{0}(\cdot)$ exists for every $\xi \in S^{N-1}$ and $\mu \geq 0$, where $a_{0}(x)=f(x, 0)$.

Let

$$
X=\left\{u \in C\left(\mathbb{R}^{N}, \mathbb{R}\right) \mid u \text { is uniformly continuous on } \mathbb{R}^{N}, \sup _{x \in \mathbb{R}^{N}}|u(x)|<\infty\right\}
$$

with norm $\|u\|_{X}=\sup _{x \in \mathbb{R}^{N}}|u(x)|$, and

$$
X^{+}=\left\{u \in X \mid u(x) \geq 0 \quad \forall x \in \mathbb{R}^{N}\right\} .
$$

For any $u_{0} \in X$, let $u\left(t, x ; u_{0}\right)$ be the solution of (1.1) with $u\left(0, x ; u_{0}\right)=u_{0}(x)$ (see section 2 for the discussions on local existence of $\left.u\left(t, x ; u_{0}\right)\right)$. If $u_{0} \in X^{+}$, then $u\left(t, x ; u_{0}\right)$ exists for all $t \geq 0$ (see Proposition 2.1).

Note that assuming (H1), (1.1) has at most one positive stationary solution in $X_{P}^{+}$(see Theorem C). If (1.1) has a positive stationary solution $u^{+}(\cdot) \in X_{p}^{+}$, let

$$
u_{\text {inf }}^{+}=\inf _{x \in \mathbb{R}^{N}} u^{+}(x)
$$

For a given $\xi \in S^{N-1}$, let

$$
\begin{array}{r}
X^{+}(\xi)=\left\{u \in X^{+} \mid \sup _{x \in \mathbb{R}^{N}} u(x)<u_{\text {inf }}^{+} \quad \liminf _{r \rightarrow-\infty} \inf _{x \cdot \xi \leq r} u(x)>0,\right. \\
\left.u(x)=0 \text { for } x \in \mathbb{R}^{N} \text { with } x \cdot \xi \gg 1\right\} .
\end{array}
$$

Definition 1.2. Assume that (1.1) has a positive stationary solution $u^{+}(x)$ which is stable with respect to perturbations in $X_{p}$ and that $\xi \in S^{N-1}$. We call a number $c^{*}(\xi) \in \mathbb{R}$ the spreading speed of (1.1) in the direction of $\xi$ if for every $u_{0} \in X^{+}(\xi)$,

$$
\liminf _{t \rightarrow \infty} \inf _{x \cdot \xi \leq c t}\left(u\left(t, x ; u_{0}\right)-u^{+}(x)\right)=0 \quad \forall c<c^{*}(\xi)
$$

and

$$
\limsup _{t \rightarrow \infty} \sup _{x \cdot \xi \geq c t} u\left(t, x ; u_{0}\right)=0 \quad \forall c>c^{*}(\xi) .
$$


Among those, the following theorem is proved in [41].

Theorem A. Assume (H1)-(H3). Assume also that $k(\cdot)$ is symmetric. Then

(1) (1.1) has a positive stable stationary solution $u^{+}(\cdot) \in X_{p}^{+}$;

(2) the spreading speed $c^{*}(\xi)$ of (1.1) in the direction of $\xi \in S^{N-1}$ exists for every $\xi \in S^{N-1}$ and

$$
c^{*}(\xi)=\inf _{\mu>0} \frac{\lambda\left(\xi, \mu, a_{0}\right)}{\mu} ;
$$

(3) $c^{*}(\xi)=c^{*}(-\xi)$ for every $\xi \in S^{N-1}$.

Regarding the assumption (H3), we make the following assumption:

(H4) $a(\cdot) \in C^{N}\left(\mathbb{R}^{N}\right) \cap X_{p}$ and the partial derivatives of $a(x)$ up to order $N-1$ at some $x_{0}$ are zero, where $x_{0}$ is such that $a\left(x_{0}\right)=\max _{x \in \mathbb{R}^{N}} a(x)$.

The following theorem is also proved in [41.

Theorem B. Assume that $k(\cdot)$ is symmetric.

(1) If $k(x)=\frac{1}{\delta^{N}} \tilde{k}\left(\frac{x}{\delta}\right)$ for all $x \in \mathbb{R}^{N}$ and $\delta>0$ is sufficiently small, where $\tilde{k}(\cdot)$ satisfies that $\tilde{k}(z)>0$ for $\|z\|<1, \tilde{k}(z)=0$ for $\|z\| \geq 1$, and $\int_{\mathbb{R}^{N}} \tilde{k}(z) d z=$ 1 , then the principal eigenvalue $\lambda(\xi, \mu, a)$ of (1.10) exists for all $\xi \in S^{N-1}$ and $\mu \in \mathbb{R}$.

(2) If $a(x)$ satisfies that $\max _{x \in \mathbb{R}^{N}} a(x)-\min _{x \in \mathbb{R}^{N}} a(x)<1$, then the conclusion in (1) holds.

(3) If (H4) is satisfied, then the conclusion in (1) holds.

We remark that when $k(\cdot)$ is not symmetric, similar results to Theorem B hold. For example, the following theorem follows from the arguments similar to those in [41.

Theorem B'. (1) If $k(x)=\frac{1}{\delta^{N}} \tilde{k}\left(\frac{x}{\delta}\right)$ for all $x \in \mathbb{R}^{N}$ and $\delta>0$ is sufficiently small, where $\tilde{k}(\cdot)$ is as in Theorem $\mathrm{B}(1)$, then the principal eigenvalue $\lambda(\xi, \mu, a)$ of (1.10) exists for all $\xi \in S^{N-1}$ and $\mu \in \mathbb{R}$.

(2) If $a(x)$ satisfies $\max _{x \in \mathbb{R}^{N}} a(x)-\min _{x \in \mathbb{R}^{N}} a(x)<\inf _{\xi \in S^{N-1}} \int_{z \cdot \xi \leq 0} k(z) d z$, then the conclusion in (1) holds.

(3) If (H4) is satisfied, then the conclusion in (1) holds.

Theorems B and $\mathrm{B}^{\prime}$ reveal such an important fact: a nonlocal dispersal operator possesses a similar principal eigenvalue theory to a random dispersal operator for the following cases: the nonlocal dispersal is nearly local; the periodic habitat is nearly globally homogeneous or it is nearly homogeneous in a region where it is most conducive to population growth in the zero-limit population.

The principal eigenvalue and principal eigenfunction theory provides an important tool to prove Theorem A. As is seen in [41, in general, (H3) may not be satisfied, which does not occur for random dispersal operators. In practice, the convolution kernel $k(\cdot)$ may not be symmetric either. It is important to see whether the spatial spreading feature is generic for monostable nonlocal KPP equations in the sense that assuming (H1) and (H2), (1.1) possesses a positive stable stationary solution $u^{+} \in X_{p}$ and a spreading speed $c^{*}(\xi)$ in every direction of $\xi \in S^{N-1}$ no matter if $k(\cdot)$ is symmetric or not and no matter if (H3) is satisfied or not. In this 
paper, we show that the spatial spreading feature is generic for general nonlocal monostable equations in the above sense. More precisely, we prove

Theorem $\mathbf{C}$ (Existence, uniqueness, and stability of positive stationary solutions).

(1) Assume (H1). (1.1) has at most one positive stationary solution $u^{+}(\cdot)$ in $X_{p}^{+}$. If there is a positive stationary solution $u^{+}(\cdot) \in X_{p}^{+}$, it is globally asymptotically stable with respect to perturbations in $X_{p}^{+} \backslash\{0\}$.

(2) If (H1) and (H2) hold, then (1.1) has exactly two stationary solutions in $X_{p}^{+}, u^{-} \equiv 0$, which is linearly unstable, and $u^{+}(\cdot) \in X_{p}^{+} \backslash\{0\}$, which is globally asymptotically stable with respect to perturbations in $X_{p}^{+} \backslash\{0\}$.

(3) $\lambda_{0}\left(a_{0}\right) \geq \lambda_{0}\left(\bar{a}_{0}\right)=\bar{a}_{0}:=\frac{1}{p_{1} p_{2} \cdots p_{N}} \int_{D} a_{0}(x) d x$, where $a_{0}(x)=f(x, 0)$ and $D=\left[0, p_{1}\right] \times\left[0, p_{2}\right] \times \cdots \times\left[0, p_{N}\right]$. If $\bar{a}_{0}>0$ and $(\mathrm{H} 1)$ is satisfied, then (H2) is satisfied and the conclusions in (2) hold.

Theorem D (Existence and variational principle of spreading speeds). Assume (H1) and (H2). Then the following hold:

(1) for any $\xi \in S^{N-1}$, (1.1) has a spreading speed $c^{*}(\xi)$ in the direction of $\xi$ and

$$
c^{*}(\xi)=\inf _{\mu>0} \frac{\lambda_{0}\left(\xi, \mu, a_{0}\right)}{\mu} ;
$$

(2) $c^{*}(\xi) \geq \bar{c}^{*}(\xi)$ for every $\xi \in S^{N-1}$, where $\bar{c}^{*}(\xi)=\inf _{\mu>0} \frac{\lambda_{0}\left(\xi, \mu, \bar{a}_{0}\right)}{\mu}$.

Theorem E (Spreading features of spreading speeds). Assume (H1) and (H2).

(1) If $u_{0} \in X^{+}$satisfies that $u_{0}(x)=0$ for $x \in \mathbb{R}^{N}$ with $|x \cdot \xi| \gg 1$, then for each $c>\max \left\{c^{*}(\xi), c^{*}(-\xi)\right\}, \lim \sup _{t \rightarrow \infty} \sup _{|x \cdot \xi| \geq c t} u\left(t, x ; u_{0}\right)=0$.

(2) Assume that $\xi \in S^{N-1}$ and $0<c<\min \left\{c^{*}(\xi), c^{*}(-\xi)\right\}$. Then for any $\sigma>0$ and $r>0, \liminf _{t \rightarrow \infty} \inf _{|x \cdot \xi| \leq c t}\left(u\left(t, x ; u_{0}\right)-u^{+}(x)\right)=0$ for every $u_{0} \in X^{+}$satisfying $u_{0}(x) \geq \sigma$ for all $x \in \mathbb{R}^{N}$ with $|x \cdot \xi| \leq r$.

(3) If $u_{0} \in X^{+}$satisfies that $u_{0}(x)=0$ for $x \in \mathbb{R}^{N}$ with $\|x\| \gg 1$, then $\lim \sup _{t \rightarrow \infty} \sup _{\|x\| \geq c t} u\left(t, x ; u_{0}\right)=0$ for all $c>\sup _{\xi \in S^{N-1}} c^{*}(\xi)$.

(4) Assume that $0<c<\inf _{\xi \in S^{N-1}}\left\{c^{*}(\xi)\right\}$. Then for any $\sigma>0$ and $r>0$, $\liminf _{t \rightarrow \infty} \inf _{\|x\| \leq c t}\left(u\left(t, x ; u_{0}\right)-u^{+}(x)\right)=0$ for every $u_{0} \in X^{+}$satisfying $u_{0}(x) \geq \sigma$ for $x \in \mathbb{R}^{N}$ with $\|x\| \leq r$.

Theorems $\mathrm{C}-\mathrm{E}$ generalize most of the results in [41] to general nonlocal monostable equations. Theorem $\mathrm{E}$ also improves the results in [41, Theorems D and E] in the sense that $r$ in Theorem E (2) and (4) can be chosen to be independent of $\sigma$. As pointed out in [41, the theories established in [28, 29, 44, 45] for spatial spreading speeds of general monostable systems cannot be applied to (1.1) due to the lack of the compactness of the solution operators of (1.1) and (1.12). The main techniques to be used in proving Theorems $\mathrm{C}-\mathrm{E}$ are the principal eigenvalue theory and spatial spreading speed theory established in [41. It should be pointed out that similar statements to Theorem $\mathrm{C}(2)$ are proved in [10 for time-independent nonlocal KPP equations. We learned about the work [10] when the current paper was almost finished. For completeness, we provide a proof of Theorem $\mathrm{C}(2)$ in the paper, which is different from the proof in [10.

Observe that Theorem $\mathrm{D}(2)$ indicates that the spatial variation cannot slow down the spatial spreading and is proved in [19] when $k(\cdot)$ is symmetric. We will study the existence of traveling wave solutions of (1.1) connecting $u^{+}$and $u^{-}$in [42]. 
The rest of the paper is organized as follows. In section 2, we present some preliminary propositions to be used in later sections. We study the positive stationary solutions of (1.1) and prove Theorem $\mathrm{C}$ in section 3. In section 4, we investigate the spreading speeds of (1.1) and prove Theorems D and E.

\section{Preliminary}

In this section, we collect some basic properties of solutions of equation (1.1) and some related nonlocal linear evolution equations to be used in later sections.

Let $X_{p}$ and $X$ be as in (1.2) and (1.14), respectively. Consider equation (1.1) and the following nonlocal linear evolution equation:

$$
\frac{\partial u}{\partial t}=\int_{\mathbb{R}^{N}} e^{-\mu(y-x) \cdot \xi} k(y-x) u(t, y) d y-u(t, x)+a(x) u(t, x), \quad x \in \mathbb{R}^{N},
$$

where $\mu \in \mathbb{R}, \xi \in S^{N-1}$, and $a \in X_{p}$. Note that if $\mu=0$ and $a(x)=a_{0}(x)(:=$ $f(x, 0))$, (2.1) reduces to (1.12), i.e., the linearization of (1.1) at $u \equiv 0$.

It follows from the general linear semigroup theory (see [18] or 37]) that for every $u_{0} \in X$, (2.1) has a unique solution $u\left(t, \cdot ; u_{0}, \xi, \mu, a\right) \in X$ with $u\left(0, x ; u_{0}, \xi, \mu, a\right)=$ $u_{0}(x)$. Put

$$
\Phi(t ; \xi, \mu, a) u_{0}=u\left(t, \cdot ; u_{0}, \xi, \mu, a\right) .
$$

Note that if $u_{0} \in X_{p}$, then $\Phi(t ; \xi, \mu, a) u_{0} \in X_{p}$ for $t \geq 0$.

By general nonlinear semigroup theory (see [18] or [37]), (1.1) has a unique (local) solution $u\left(t, x ; u_{0}\right)$ with $u\left(0, x ; u_{0}\right)=u_{0}(x)$ for every $u_{0} \in X$. Also if $u_{0} \in X_{p}$, then $u\left(t, x ; u_{0}\right) \in X_{p}$ for $t$ in the existence interval of the solution $u\left(t, x ; u_{0}\right)$.

Throughout this section, we assume that $\xi \in S^{N-1}$ and $\mu \in \mathbb{R}$ are fixed, unless otherwise specified.

2.1. Comparison principle and monotonicity. Let $X_{p}^{+}$and $X^{+}$be as in (1.3) and (1.15), respectively. Let

$$
\operatorname{Int}\left(X_{p}^{+}\right)=\left\{v \in X_{p} \mid v(x)>0, x \in \mathbb{R}^{N}\right\} .
$$

For $v_{1}, v_{2} \in X_{p}$, we define

$$
\begin{gathered}
v_{1} \leq v_{2} \quad\left(v_{1} \geq v_{2}\right) \quad \text { if } \quad v_{2}-v_{1} \in X_{p}^{+} \quad\left(v_{1}-v_{2} \in X_{p}^{+}\right), \\
v_{1} \ll v_{2} \quad\left(v_{1} \gg v_{2}\right) \quad \text { if } \quad v_{2}-v_{1} \in \operatorname{Int}\left(X_{p}^{+}\right) \quad\left(v_{1}-v_{2} \in \operatorname{Int}\left(X_{p}^{+}\right)\right) .
\end{gathered}
$$

For $u_{1}, u_{2} \in X$, we define

$$
u_{1} \leq u_{2} \quad\left(u_{1} \geq u_{2}\right) \quad \text { if } \quad u_{2}-u_{1} \in X^{+} \quad\left(u_{1}-u_{2} \in X^{+}\right) .
$$

A continuous function $u(t, x)$ on $[0, T) \times \mathbb{R}^{N}$ is called a super-solution or subsolution of (2.1) if $\frac{\partial u}{\partial t}$ exists and is continuous on $[0, T) \times \mathbb{R}^{N}$ and satisfies

or

$$
\frac{\partial u}{\partial t} \geq \int_{\mathbb{R}^{N}} e^{-\mu(y-x) \cdot \xi} k(y-x) u(t, y) d y-u(t, x)+a(x) u(t, x), \quad x \in \mathbb{R}^{N}
$$

$$
\frac{\partial u}{\partial t} \leq \int_{\mathbb{R}^{N}} e^{-\mu(y-x) \cdot \xi)} k(y-x) u(t, y) d y-u(t, x)+a(x) u(t, x), \quad x \in \mathbb{R}^{N}
$$

for $t \in(0, T)$. Super-solutions and sub-solutions of (1.1) are defined in an analogous way. 
Proposition 2.1 (Comparison principle).

(1) If $u_{1}(t, x)$ and $u_{2}(t, x)$ are the sub-solution and super-solution of (2.1) on $[0, T)$, respectively, $u_{1}(0, \cdot) \leq u_{2}(0, \cdot)$, and $u_{2}(t, x)-u_{1}(t, x) \geq-\beta_{0}$ for $(t, x) \in[0, T) \times \mathbb{R}^{N}$ and some $\beta_{0}>0$, then $u_{1}(t, \cdot) \leq u_{2}(t, \cdot)$ for $t \in[0, T)$.

(2) If $u_{1}(t, x)$ and $u_{2}(t, x)$ are bounded sub- and super-solutions of (1.1) on $[0, T)$, respectively, and $u_{1}(0, \cdot) \leq u_{2}(0, \cdot)$, then $u_{1}(t, \cdot) \leq u_{2}(t, \cdot)$ for $t \in$ $[0, T)$.

(3) For every $u_{0} \in X^{+}, u\left(t, x ; u_{0}\right)$ exists for all $t \geq 0$.

Proof. The proof follows from the arguments in [41, Proposition 2.1].

Proposition 2.2 (Strong monotonicity). Suppose that $u_{1}, u_{2} \in X_{p}$ and $u_{1} \leq u_{2}$.

(1) If $u_{1} \neq u_{2}$, then $\Phi(t ; \xi, \mu, a) u_{1} \ll \Phi(t ; \xi, \mu, a) u_{2}$ for all $t>0$.

(2) If $u_{1} \neq u_{2}$, then $u\left(t, \cdot ; u_{1}\right) \ll u\left(t, \cdot ; u_{2}\right)$ for every $t>0$ at which both $u\left(t, \cdot ; u_{1}\right)$ and $u\left(t, \cdot ; u_{2}\right)$ exist.

Proof. The proof follows from the arguments in [41, Proposition 2.2].

For given $\rho \geq 0$, let

$$
X(\rho)=\left\{u \in C\left(\mathbb{R}^{N}, \mathbb{R}\right) \mid \text { the function } x \mapsto e^{-\rho\|x\|} u(x) \text { belongs to } X\right\}
$$

equipped with the norm $\|u\|_{X(\rho)}=\sup _{x \in \mathbb{R}^{N}} e^{-\rho\|x\|}|u(x)|$.

Remark 2.1. For every $u_{0} \in X(\rho)(\rho \geq 0)$, (2.1) has a unique solution $u\left(t, \cdot ; u_{0}, \xi, \mu\right)$ $\in X(\rho)$ with $u\left(0, x ; u_{0}, \xi, \mu\right)=u_{0}(x)$. Moreover, Proposition 2.1 holds for such solutions of (2.1).

2.2. Principal eigenvalue. Throughout this subsection, $X_{p}$ is as in (1.2), $a \in X_{p}$, and $a_{\max }=\max _{x \in \mathbb{R}^{N}} a(x), a_{\min }=\min _{x \in \mathbb{R}^{N}} a(x) . a(\cdot) I: X_{p} \rightarrow X_{p}$ has the same meaning as in (1.5) with $a_{0}(\cdot)$ being replaced by $a(\cdot)$ and $K_{\xi, \mu}: X_{p} \rightarrow X_{p}$ is understood as in (1.11), $\xi \in S^{N-1}$, and $\mu \in \mathbb{R}$.

Proposition 2.3. If $a(x)$ is a constant function, then $\lambda(\xi, \mu, a)$ exists and

$$
\lambda(\xi, \mu, a)=\int_{\mathbb{R}^{N}} e^{-\mu y \cdot \xi} k(y) d y-1+a .
$$

Moreover, there is $M_{0}>0$ such that $\lambda(\mu, \xi, a) \geq M_{0} \mu^{2}$ for $\mu \gg 1, \xi \in S^{N-1}$.

Proof. Note that $K_{\xi, \mu}: X_{p} \rightarrow X_{p}$ is a compact and positive operator and $K_{\xi, \mu}^{n}$ is strongly positive for some $n \geq 1$. Note also that $\lambda=\int_{\mathbb{R}^{N}} e^{-\mu y \cdot \xi} k(y) d y-1+a$ is an eigenvalue of $K_{\xi, \mu}-I+a(\cdot) I$ with an eigenfunction $\phi(x) \equiv 1$. Then by the Krein-Rutman theorem, $\lambda(\xi, \mu, a)$ exists and $\lambda(\mu, \xi, a)=\int_{\mathbb{R}^{N}} e^{-\mu y \cdot \xi} k(y) d y-1+a$.

By the fact that $k(x)>0$ for $\|x\|<\delta$, there are $\epsilon_{0}, \delta_{0}>0$ such that

$$
k(x) \geq \epsilon_{0} \quad \text { for } \quad\|x\| \leq \delta_{0} .
$$

This implies that

$$
\begin{aligned}
\lambda(\xi, \mu, a) & \geq \epsilon_{0} \int_{\|x\| \leq \delta_{0}} e^{-\mu x \cdot \xi} d x-1+a \\
& =\epsilon_{0} \int_{\|x\| \leq \delta_{0}}\left(1+\frac{\mu^{2}(x \cdot \xi)^{2}}{2 !}+\frac{\mu^{4}(x \cdot \xi)^{4}}{4 !}+\cdots\right) d x-1+a \\
& \geq M_{0} \mu^{2}
\end{aligned}
$$

for $\mu \gg 1$ and $\xi \in S^{N-1}$, where $M_{0}=\inf _{\xi \in S^{N-1}} \frac{\epsilon_{0} \int_{\|x\| \leq \delta_{0}}(x \cdot \xi)^{2} d x}{2}$. 
Proposition 2.4. If a satisfies (H4), then the principal eigenvalue $\lambda(\xi, \mu, a)$ of (1.10) exists for all $\xi \in S^{N-1}$ and $\mu \in \mathbb{R}$.

Proof. The proof follows from the arguments of [41, Theorem B].

Let $D=\left[0, p_{1}\right] \times\left[0, p_{2}\right] \times \cdots \times\left[0, p_{N}\right]$ and for given $a \in X_{p}$, let

$$
\bar{a}=\frac{1}{p_{1} p_{2} \cdots p_{N}} \int_{D} a(x) d x .
$$

Proposition 2.5. If $a \in X_{p}$ satisfies (H3), then $\lambda(\xi, \mu, a) \geq \lambda(\xi, \mu, \bar{a})$.

Proof. The proof follows from the arguments of [19, Theorem 2.1].

Proposition 2.6. Given $a \in X_{p}, \lambda_{0}(\xi, \mu, a)=\ln r(\Phi(1 ; \xi, \mu, a))$ for all $\xi \in S^{N-1}$ and $\mu \in \mathbb{R}$.

Proof. The proof follows from the spectral theorem for bounded linear operators (see [37]).

\section{Positive Stationary solutions}

In this section, we investigate the existence, uniqueness, and stability of positive equilibrium of (1.1) and prove Theorem C.

Suppose that $u=u^{*}$ is an equilibrium solution of (1.1) in $X_{p}^{+} \backslash\{0\} . u=u^{*}$ is said to be globally asymptotically stable in $X_{p}^{+} \backslash\{0\}$ if for any $u_{0} \in X_{p}^{+} \backslash\{0\}$, $u\left(t, \cdot ; u_{0}\right) \rightarrow u^{*}$ in $X_{p}$ as $t \rightarrow \infty$.

As mentioned in the introduction, Theorem $\mathrm{C}(2)$ follows from the results and arguments in 10. For completeness, we provide a proof. We first prove two lemmas, which will also be used to prove Theorem $\mathrm{D}$ in the next section.

Lemma 3.1. Suppose that $\left\{a_{n}\right\},\left\{a^{n}\right\} \subset X_{p}$ satisfy

$$
a_{n}(\cdot) \leq a(\cdot) \leq a^{n}(\cdot) \quad \text { for } n \geq 1 \quad \text { and } \quad\left\|a_{n}-a^{n}\right\|_{X_{p}} \rightarrow 0 \quad \text { as } n \rightarrow \infty .
$$

Then for any $\xi \in S^{N-1}$ and $\mu \in \mathbb{R}$,

$$
\lambda_{0}\left(\xi, \mu, a_{n}\right) \leq \lambda_{0}(\xi, \mu, a) \leq \lambda_{0}\left(\xi, \mu, a^{n}\right) \quad \text { for } \quad n \geq 1
$$

and

$$
\lambda_{0}\left(\xi, \mu, a_{n}\right)-\lambda_{0}\left(\xi, \mu, a^{n}\right) \rightarrow 0 \quad \text { as } \quad n \rightarrow \infty .
$$

Proof. By Propositions 2.1 and 2.2.

$$
r\left(\Phi\left(1 ; \xi, \mu, a_{n}\right)\right) \leq r(\Phi(1 ; \xi, \mu, a)) \leq r\left(\Phi\left(1 ; \xi, \mu, a^{n}\right)\right) \quad \forall n \geq 1, \xi \in S^{N-1}, \mu \in \mathbb{R} .
$$

This together with Proposition 2.6 implies (3.1). By (3.1), for any $\xi \in S^{N-1}, \mu \in \mathbb{R}$, and $\epsilon>0$,

$$
\lambda_{0}(\xi, \mu, a-\epsilon) \leq \lambda_{0}\left(\xi, \mu, a_{n}\right) \leq \lambda_{0}(\xi, \mu, a) \leq \lambda_{0}\left(\xi, \mu, a^{n}\right) \leq \lambda_{0}(\xi, \mu, a+\epsilon)
$$

for $n \gg 1$. This together with $\lambda_{0}(\xi, \mu, a \pm \epsilon)=\lambda_{0}(\xi, \mu, a) \pm \epsilon$ implies (3.2).

Lemma 3.2. Given $a \in X_{p}, \lambda_{0}(\xi, \mu, a) \geq \lambda_{0}(\xi, \mu, \bar{a})$ for any $\xi \in S^{N-1}$ and $\mu \in \mathbb{R}$.

Proof. Take $a_{n} \in C^{N}\left(\mathbb{R}^{N}\right) \cap X_{p}$ such that $a_{n}$ satisfies (H4) and

$$
a_{n}(\cdot) \leq a(\cdot) \quad \text { for } n \geq 1 \text { and }\left\|a_{n}-a\right\|_{X_{p}} \rightarrow 0 \text { as } n \rightarrow \infty .
$$

By Proposition 2.4, $\lambda\left(\xi, \mu, a_{n}\right)$ exists and $\lambda\left(\xi, \mu, a_{n}\right)=\lambda_{0}\left(\xi, \mu, a_{n}\right)$ for $n \geq 1$. By Proposition 2.5. $\lambda_{0}\left(\xi, \mu, a_{n}\right) \geq \lambda_{0}\left(\xi, \mu, \bar{a}_{n}\right)$ for $n \geq 1$. The lemma follows by letting $n \rightarrow \infty$ and applying Lemma 3.1 . 
Proof of Theorem C. (1) This follows from the arguments in [26, Lemma 3.3].

(2) Let $a_{0}(x)=f(x, 0)$ and $0<\delta_{0}<\epsilon_{0}$ be such that

$$
\lambda_{0}-2 \epsilon_{0}>0 \text { and } f(x, u) \geq a(x, 0)-\epsilon_{0} \quad \text { for } \quad 0<u<\delta_{0} .
$$

Take $a_{n} \in C^{N}\left(\mathbb{R}^{N}\right) \cap X_{p}$ such that $a_{n}$ satisfies (H4) and

$$
a_{n}(\cdot) \leq a_{0}(\cdot) \text { for } n \geq 1 \text { and }\left\|a_{n}-a_{0}\right\|_{X_{p}} \rightarrow 0 \text { as } n \rightarrow \infty \text {. }
$$

Then by Lemma 3.1,

$$
\lambda_{0}\left(a_{n}\right) \leq \lambda_{0}\left(a_{0}\right) \quad \forall n \geq 1 \quad \text { and } \quad \lambda_{0}\left(a_{n}\right)-\lambda_{0}\left(a_{0}\right) \rightarrow 0 \quad \text { as } \quad n \rightarrow \infty .
$$

This implies that

$$
\lambda_{0}\left(a_{n}\right)-2 \epsilon_{0}>0 \text { for } n \gg 1 .
$$

Note that

$$
a_{n}(x)-\epsilon_{0}-u \geq a_{n}(x)-2 \epsilon_{0} \quad \text { for } \quad 0<u<\delta_{0} .
$$

Hence

$$
u f(x, u) \geq u\left(a_{n}(x)-2 \epsilon_{0}\right) \quad \text { for } \quad 0<u<\delta_{0} .
$$

Let $\phi_{n}(x) \in X_{p}^{+}$be the eigenfunction of $K-I+a_{n}(\cdot) I$ associated to $\lambda_{0}\left(a_{n}\right)$ with $\left\|\phi_{n}\right\|_{X_{p}}=1$. Let $b_{0}>0$ with $b_{0} e^{\lambda_{0}\left(a_{n}\right)-2 \epsilon_{0}}<\delta_{0}$ and $u_{0}=b_{0} \phi_{n}$. By Proposition 2.1.

$$
u\left(t, \cdot ; u_{0}\right) \geq b_{0} e^{\left(\lambda_{0}\left(a_{n}\right)-2 \epsilon_{0}\right) t} \phi_{n}(\cdot)>u_{0}(\cdot) \quad \text { for } \quad 0<t<1 .
$$

It then follows that $u\left(t, \cdot ; u_{0}\right)$ is monotonically increasing as $t$ is increasing. Let $u^{+}(x)=\lim _{t \rightarrow \infty} u\left(t, x ; u_{0}\right)$. By the arguments in [26, Theorem 3.2] or the arguments in [2, Theorem 2.6], $u^{+} \in X_{p}^{+} \backslash\{0\}$. Then by (1), $u=u^{+}$is a globally asymptotically stable stationary solution with respect to the perturbations in $X_{p}^{+}$. (2) then follows.

(3) Let $a_{n}$ be as in (2). Then by (2) and Proposition 2.5

$$
\lambda_{0}\left(a_{0}\right) \geq \lambda_{0}\left(a_{n}\right) \geq \lambda_{0}\left(\bar{a}_{n}\right)=\bar{a}_{n} .
$$

Letting $n \rightarrow \infty$, we have $\lambda_{0}\left(a_{0}\right) \geq \lambda_{0}\left(\bar{a}_{0}\right)=\bar{a}_{0}$. Therefore, if $\bar{a}_{0}>0$, then (H2) is satisfied and the conclusions in (2) hold if (H1) is also satisfied.

\section{Spreading SPEeds}

In this section, we investigate the existence and characterization of the spreading speeds of (1.1) and prove Theorems D and E.

We first recall the notion of spreading speed intervals introduced in 41 and prove some lemmas.

Definition 4.1. For a given vector $\xi \in S^{N-1}$, let

$$
C_{\mathrm{inf}}^{*}(\xi)=\left\{c \mid \forall u_{0} \in X^{+}(\xi), \liminf _{t \rightarrow \infty} \inf _{x \cdot \xi \leq c t}\left(u\left(t, x ; u_{0}\right)-u^{+}(x)\right)=0\right\}
$$

and

$$
C_{\text {sup }}^{*}(\xi)=\left\{c \mid \forall u_{0} \in X^{+}(\xi), \limsup _{t \rightarrow \infty} \sup _{x \cdot \xi \geq c t} u\left(t, x ; u_{0}\right)=0\right\} .
$$

Define

$$
c_{\mathrm{inf}}^{*}(\xi)=\sup \left\{c \mid c \in C_{\mathrm{inf}}^{*}(\xi)\right\}, \quad c_{\mathrm{sup}}^{*}(\xi)=\inf \left\{c \mid c \in C_{\mathrm{sup}}^{*}(\xi)\right\} .
$$

We call $\left[c_{\mathrm{inf}}^{*}(\xi), c_{\mathrm{sup}}^{*}(\xi)\right]$ the spreading speed interval of (1.1) in the direction of $\xi$.

Observe that $c^{*}(\xi)$ exists if and only if $c_{\text {inf }}^{*}(\xi)=c_{\text {sup }}^{*}(\xi)$. 
Lemma 4.1. Assume (H1) and (H2). For every $\xi \in S^{N-1}$, there is $\mu^{*}(\xi) \in(0, \infty)$ such that

$$
\frac{\lambda_{0}\left(\xi, \mu^{*}(\xi), a_{0}\right)}{\mu^{*}(\xi)}=\inf _{\mu>0} \frac{\lambda_{0}\left(\xi, \mu, a_{0}\right)}{\mu} .
$$

Proof. First, it is not difficult to see that $\lambda_{0}\left(\xi, \mu, a_{0}\right)$ is continuous in $\mu$. By (H2), $\lambda_{0}\left(\xi, 0, a_{0}\right)>0$ and hence $\lim _{\mu \rightarrow 0+} \frac{\lambda_{0}\left(\xi, \mu, a_{0}\right)}{\mu}=\infty$. By Propositions 2.3 and 2.5. $\lim _{\mu \rightarrow \infty} \frac{\lambda_{0}\left(\xi, \mu, a_{0}\right)}{\mu}=\infty$. The lemma then follows.

Consider the space-shifted equations of (1.1),

$$
\frac{\partial u}{\partial t}=\int_{\mathbb{R}^{N}} k(y-x) u(t, y) d y-u(t, x)+u(t, x) f(x+z, u(t, x)), \quad x \in \mathbb{R}^{N},
$$

where $z \in \mathbb{R}^{N}$. Let $u\left(t, x ; u_{0}, z\right)$ be the solution of (4.1) with $u\left(0, x ; u_{0}, z\right)=u_{0}(x)$ for $u_{0} \in X$.

Lemma 4.2. Let $\xi \in S^{N-1}, u_{0} \in X^{+}$with $\liminf _{x \cdot \xi \rightarrow-\infty} u_{0}(x)>0$ and $\lim \sup _{x \cdot \xi \rightarrow \infty} u_{0}(x)=0$, and $c \in \mathbb{R}$ be given. If there are $\delta_{0}$ and $T_{0}>0$ such that

$$
\liminf _{x \cdot \xi \leq c n T_{0}, n \rightarrow \infty} u\left(n T_{0}, x ; u_{0}, z\right) \geq \delta_{0} \quad \text { uniformly in } \quad z \in \mathbb{R}^{N}
$$

then for every $c^{\prime}<c$,

$$
\liminf _{x \cdot \xi \leq c^{\prime} t, t \rightarrow \infty}\left(u\left(t, x ; u_{0}, z\right)-u^{+}(x+z)\right)=0 \quad \text { uniformly in } \quad z \in \mathbb{R}^{N} .
$$

Proof. This follows from the arguments of [41, Proposition 4.4].

Proof of Theorem D. (1) First, we prove that $c_{\text {sup }}^{*}(\xi) \leq \inf _{\mu>0} \frac{\lambda_{0}\left(\xi, \mu, a_{0}\right)}{\mu}$.

Let $a^{n}(\cdot) \in C^{N}\left(\mathbb{R}^{N}\right) \cap X_{p}$ be such that $a^{n}$ satisfies (H4),

$$
a^{n} \geq a_{0} \quad \text { for } \quad n \geq 1 \text { and }\left\|a^{n}-a\right\|_{X_{p}} \rightarrow 0 \quad \text { as } n \rightarrow \infty .
$$

Then by Lemma 3.1 ,

$$
\lambda_{0}\left(\xi, \mu, a^{n}\right) \rightarrow \lambda_{0}\left(\xi, \mu, a_{0}\right) \quad \text { as } \quad n \rightarrow \infty .
$$

Let $\phi^{n}$ be the positive eigenfunction of $K_{\xi, \mu}-I+a^{n}(\cdot) I$ corresponding to $\lambda\left(\xi, \mu, a^{n}\right)$ $=\lambda_{0}\left(\xi, \mu, a^{n}\right)$ with $\left\|\phi^{n}\right\|_{X_{p}}=1$. Note that

$$
u f(x, u) \leq u f(x, 0) \leq a^{n}(x) u \quad \text { for } \quad x \in \mathbb{R}^{N}, u \geq 0
$$

and

$$
\left(\Phi\left(t, \xi, 0, a^{n}\right) u_{\xi, \mu}\right)(x)=e^{-\mu\left(x \cdot \xi-\frac{\lambda_{0}\left(\xi, \mu, a^{n}\right)}{\mu} t\right)} \phi^{n}(x),
$$

where $u_{\xi, \mu}(x)=e^{-\mu x \cdot \xi} \phi^{n}(x)$. Hence by Proposition 2.1 and Remark 2.1, for any $u_{0} \in X^{+}(\xi)$ and any $\mu>0$,

$$
u\left(t, x ; u_{0}\right) \leq M e^{-\mu\left(x \cdot \xi-\frac{\lambda_{0}\left(\xi, \mu, a^{n}\right)}{\mu} t\right)} \phi^{n}(x) \quad \text { for } \quad t \geq 0
$$

where $M$ is such that $u,(x) \leq M u_{\xi, \mu}(x)$. This implies that

$$
c_{\text {sup }}^{*}(\xi) \leq \frac{\lambda_{0}\left(\xi, \mu, a^{n}\right)}{\mu} \quad \forall \mu>0, n \geq 1
$$

and then

$$
c_{\text {sup }}^{*}(\xi) \leq \frac{\lambda_{0}\left(\xi, \mu, a_{0}\right)}{\mu} \quad \forall \mu>0 .
$$


Therefore,

$$
c_{\mathrm{sup}}^{*}(\xi) \leq \inf _{\mu>0} \frac{\lambda_{0}\left(\xi, \mu, a_{0}\right)}{\mu} .
$$

Next, we prove $c_{\mathrm{inf}}^{*}(\xi) \geq \inf _{\mu>0} \frac{\lambda_{0}\left(\xi, \mu, a_{0}\right)}{\mu}$. For any $\epsilon>0$, there is $\delta_{0}>0$ such that

$$
f(x, u) \geq f(x, 0)-\epsilon \text { for } \quad x \in \mathbb{R}^{N}, 0<u<\delta_{0} .
$$

Let $a_{n}(\cdot) \in C^{N}\left(\mathbb{R}^{N}\right) \cap X_{p}$ be such that $a_{n}$ satisfies (H4),

$$
f(\cdot, 0)-2 \epsilon \leq a_{n}(\cdot) \leq f(\cdot, 0)-\epsilon \quad \forall n \geq 1 .
$$

Let

$$
c_{n}^{*}(\xi)=\inf _{\mu>0} \frac{\lambda\left(\xi, \mu, a_{n}\right)}{\mu} .
$$

Applying the arguments in [41, Theorem C], there is $u_{0}(\cdot ; z) \in X^{+}(\xi)$ such that

$$
\liminf _{x \cdot \xi \rightarrow-\infty} \inf _{z \in \mathbb{R}^{N}} u_{0}(x ; z)>0
$$

and

$$
u\left(1, x ; u_{0}(\cdot ; z), z\right) \geq u_{0}\left(x-\left(c_{n}^{*}(\xi)-\epsilon\right) \xi ;\left(c_{n}^{*}(\xi)-\epsilon\right) \xi+z\right) \quad \forall z \in \mathbb{R}^{N} .
$$

This implies that

$$
u\left(m, x ; u_{0}(\cdot ; z), z\right) \geq u_{0}\left(x-m\left(c_{n}^{*}(\xi)-\epsilon\right) \xi ; m\left(c_{n}^{*}(\xi)-\epsilon\right) \xi+z\right) \quad \forall m \geq 1, z \in \mathbb{R}^{N} .
$$

Then by Lemma 4.2 ,

By Lemma 3.1 .

$$
c_{\mathrm{inf}}^{*}(\xi) \geq c_{n}^{*}(\xi)-\epsilon
$$

$$
c_{\mathrm{inf}}^{*}(\xi) \geq \inf _{\mu>0} \frac{\lambda_{0}\left(\xi, \mu, a_{0}\right)-2 \epsilon}{\mu}-\epsilon .
$$

Letting $\epsilon \rightarrow 0$, by Lemma 4.1 we have

$$
c_{\mathrm{inf}}^{*}(\xi) \geq \inf _{\mu>0} \frac{\lambda_{0}\left(\xi, \mu, a_{0}\right)}{\mu} .
$$

By (4.3) and (4.4)

$$
c_{\mathrm{sup}}^{*}(\xi)=c_{\mathrm{inf}}^{*}(\xi)=\inf _{\mu>0} \frac{\lambda_{0}\left(\xi, \mu, a_{0}\right)}{\mu} .
$$

Hence $c^{*}(\xi)$ exists and

$$
c^{*}(\xi)=\inf _{\mu>0} \frac{\lambda_{0}\left(\xi, \mu, a_{0}\right)}{\mu} .
$$

(2) This follows from (1) and Proposition 2.5 .

Proof of Theorem E. (1) Fix $c>\max \left\{c^{*}(\xi), c^{*}(-\xi)\right\}$. As in the proof of Theorem D (1), let $a^{n}(\cdot) \in C^{N}\left(\mathbb{R}^{N}\right) \cap X_{p}$ be such that $a^{n}$ satisfies (H4),

$$
a^{n} \geq a_{0} \quad \text { for } n \geq 1 \text { and }\left\|a^{n}-a\right\|_{X_{p}} \rightarrow 0 \text { as } n \rightarrow \infty .
$$

Choose $\mu>0$ and $n \gg 1$ such that

$$
\frac{\lambda_{0}\left(\xi, \mu, a^{n}\right)}{\mu}<c .
$$

Choose $M>1$ such that

$$
u_{0}(x) \leq M e^{-\mu x \cdot \xi} \phi^{n}(x),
$$


where $\phi^{n}(x)$ is the positive eigenfunction of $K_{\xi, \mu}-I+a^{n}(\cdot) I$ corresponding to $\lambda\left(\xi, \mu, a^{n}\right)=\lambda_{0}\left(\xi, \mu, a^{n}\right)$ with $\left\|\phi^{n}\right\|_{X_{p}}=1$. By similar arguments as in Theorem D $(1)$,

$$
u\left(t, x ; u_{0}\right) \leq e^{-\mu\left(x \cdot \xi-\frac{\lambda_{0}\left(\xi, \mu, a^{n}\right)}{\mu} t\right)} \phi^{n}(x) \quad \text { for } \quad t \geq 0 .
$$

This implies that

$$
\limsup _{t \rightarrow \infty} \sup _{x \cdot \xi \geq c t} u\left(t, x ; u_{0}\right)=0 .
$$

Similarly, it can be proved that

$$
\limsup _{t \rightarrow \infty} \sup _{x \cdot \xi \leq-c t} u\left(t, x ; u_{0}\right)=0 .
$$

Thus (1) follows from (4.5) and (4.6).

(2) First, following from the arguments in 41, Theorem D (2)], for each $\sigma>0$, there is $r_{\sigma}>0$ such that

$$
\liminf _{t \rightarrow \infty} \inf _{|x \cdot \xi| \leq c t}\left(u\left(t, x ; u_{0}\right)-u^{+}(x)\right)=0
$$

for every $u_{0} \in X^{+}$satisfying $u_{0}(x) \geq \sigma$ for all $x \in \mathbb{R}^{N}$ with $|x \cdot \xi| \leq r_{\sigma}$.

We claim that (2) can be proved by similar arguments as in [28, Corollary 2.16]. In fact, let $\sigma>0$ and $r>0$ be given. Suppose that $u_{0} \in X^{+}$satisfies $u_{0}(x) \geq \sigma$ for all $x \in \mathbb{R}^{N}$ with $|x \cdot \xi| \leq r$. Note that there is $m>0$ such that

$$
-1+f\left(x, u\left(t, x ; u_{0}\right)\right) \geq-m \quad \forall x \in \mathbb{R}^{N}, t \geq 0 .
$$

Then

and hence

$$
u_{t}\left(t, x ; u_{0}\right) \geq \int_{\mathbb{R}^{N}} k(y-x) u\left(t, y ; u_{0}\right) d y-m u\left(t, x ; u_{0}\right),
$$

$$
\left(e^{m t} u\left(t, x ; u_{0}\right)\right)_{t} \geq \int_{\mathbb{R}^{N}} k(y-x) e^{m t} u\left(t, y ; u_{0}\right) d y .
$$

This together with Proposition 2.1 implies that

$$
e^{m t} u\left(t, \cdot ; u_{0}\right) \geq e^{K t} u_{0},
$$

where $e^{K t}=I+K t+\frac{K^{2} t^{2}}{2 !}+\cdots$ and $K u$ is defined as in (1.4) with $u \in X_{p}$ being replaced by $u \in X$. It is then not difficult to see that there is $\rho \in(0,1)$ such that

$$
\rho \sigma<\inf _{x \in \mathbb{R}^{N}} u^{+}(x) \quad \text { and } \quad u\left(1, x ; u_{0}\right) \geq \rho \sigma \quad \text { for } \quad|x \cdot \xi| \leq r_{\sigma} .
$$

Let $v_{0}(x)=\frac{1}{\rho} u\left(1, x ; u_{0}\right)$. Then by (4.7),

$$
\liminf _{t \rightarrow \infty} \inf _{|x \cdot \xi| \leq c t}\left(u\left(t, x ; v_{0}\right)-u^{+}(x)\right)=0 .
$$

By (H2) and Proposition 2.1, we have

$$
u\left(t+1, x ; u_{0}\right) \equiv u\left(t, x ; \rho v_{0}\right) \geq \rho u\left(t, x ; v_{0}\right) .
$$

By (4.8) and (4.9), there is $T>0$ such that

$$
u\left(T, x ; u_{0}\right) \geq \rho \sigma \quad \text { for } \quad|x \cdot \xi| \leq r_{\rho \sigma} .
$$

By (4.7) and (4.10),

$$
\liminf _{t \rightarrow \infty} \inf _{|x \cdot \xi| \leq c t}\left(u\left(t+T, x ; u_{0}\right)-u^{+}(x)\right)=0 .
$$

Then (2) follows from the arbitrariness of $c$ with $0<c<\min \left\{c^{*}(\xi), c^{*}(-\xi)\right\}$.

(3) This can be proved by the arguments in (1) and in [41, Theorem E (1)]. 
(4) This can be proved by the arguments in (2) and in [41, Theorem E (2)].

\section{ACKNOWLEDGEMENT}

The authors would like to thank the referee for valuable comments and suggestions.

\section{REFERENCES}

[1] D. G. Aronson and H. F. Weinberger, Multidimensional nonlinear diffusions arising in population genetics, Adv. Math. 30 (1978), pp. 33-76. MR.511740 (80a:35013)

[2] P. Bates and G. Zhao, Existence, uniqueness and stability of the stationary solution to a nonlocal evolution equation arising in population dispersal, J. Math. Anal. Appl. 332 (2007), pp. 428-440. MR2319673 (2008h:35019)

[3] H. Berestycki, F. Hamel, and N. Nadirashvili, The speed of propagation for KPP type problems, I - Periodic framework, J. Eur. Math. Soc. 7 (2005), pp. 172-213. MR.2127993 (2005k:35186)

[4] H. Berestycki, F. Hamel, and N. Nadirashvili, The speed of propagation for KPP type problems, II - General domains, J. Amer. Math. Soc. 23 (2010), no. 1, pp. 1-34. MR 2552247 (2010k:35260)

[5] H. Berestycki, F. Hamel, and L. Roques, Analysis of periodically fragmented environment model: II - Biological invasions and pulsating traveling fronts, J. Math. Pures Appl. 84 (2005), pp. 1101-1146. MR2155900 (2006d:35123)

[6] Reinhard Bürger, Perturbations of positive semigroups and applications to population genetics, Math. Z. 197 (1988), pp. 259-272. MR923493 (89b:47057)

[7] E. Chasseigne, M. Chaves, and J. D. Rossi, Asymptotic behavior for nonlocal diffusion equations, J. Math. Pures Appl. 86 (2006), 271-291. MR2257732 (2007e:35279)

[8] C. Cortazar, M. Elgueta, and J. D. Rossi, Nonlocal diffusion problems that approximate the heat equation with Dirichlet boundary conditions, Israel J. of Math. 170 (2009), pp. 53-60. MR2506317 (2010e:35197)

[9] J. Coville, On uniqueness and monotonicity of solutions of non-local reaction diffusion equation, Annali di Matematica 185(3) (2006), pp. 461-485. MR2231034(2007e:35156)

[10] J. Coville, On a simple criterion for the existence of a principal eigenfunction of some nonlocal operators, J. Differential Equations 249 (2010), pp. 2921-2953. MR2718672

[11] J. Coville and L. Dupaigne, Propagation speed of travelling fronts in non local reactiondiffusion equations, Nonlinear Analysis 60 (2005), pp. 797-819. MR2113158 (2005k:35213)

[12] J. Coville, J. Dávila, and S. Martínez, Existence and uniqueness of solutions to a nonlocal equation with monostable nonlinearity, SIAM J. Math. Anal. 39 (2008), pp. 1693-1709. MR2377295 (2009d:45013)

[13] P. C. Fife and J. B. McLeod, The approach of solutions of nonlinear diffusion equations to travelling front solutions, Arch. Ration. Mech. Anal. 65 (1977), pp. 335-361. MR0442480 $(56: 862)$

[14] R. Fisher, The wave of advance of advantageous genes, Ann. of Eugenics 7 (1937), pp. 335369.

[15] J. García-Melán and J. D. Rossi, On the principal eigenvalue of some nonlocal diffusion problems, J. Differential Equations 246 (2009), pp. 21-38. MR2467013 (2009j:35150)

[16] M. Grinfeld, G. Hines, V. Hutson, K. Mischaikow, and G. T. Vickers, Non-local dispersal, Differential Integral Equations 18 (2005), pp. 1299-1320. MR.2174822 (2006m:35033)

[17] F. Hamel, Qualitative properties of monostable pulsating fronts: exponential decay and monotonicity, J. Math. Pures Appl. 89 (2008), pp. 355-399. MR2401143 (2009g:35132)

[18] D. Henry, Geometric Theory of Semilinear Parabolic Equations, Lecture Notes in Math. 840, Springer-Verlag, Berlin, 1981. MR610244 (83j:35084)

[19] G. Hetzer, W. Shen, and A. Zhang, Effects of spatial variations and dispersal strategies on principal eigenvalues of dispersal operators and spreading speeds of monostable equations, Rocky Mountain Journal of Mathematics, to appear.

[20] J. Huang and W. Shen, Speeds of spread and propagation for KPP models in time almost and space periodic media, SIAM J. Appl. Dynam. Syst. 8 (2009), pp. 790-821. MR 2533625 (2010h:35192) 
[21] W. Hudson and B. Zinner, Existence of traveling waves for reaction diffusion equations of Fisher type in periodic media. Boundary value problems for functional-differential equations, 187-199, World Sci. Publ., River Edge, NJ, 1995. MR.1375475|(97a:35112)

[22] V. Hutson and M. Grinfeld, Non-local dispersal and bistability, Euro. J. Appl. Math. 17 (2006), pp. 221-232. MR 2266484 (2007j:35087)

[23] V. Hutson, S. Martinez, K. Mischaikow, and G.T. Vickers, The evolution of dispersal, J. Math. Biol. 47 (2003), pp. 483-517. MR2028048 (2004j:92074)

[24] V. Hutson, W. Shen and G.T. Vickers, Spectral theory for nonlocal dispersal with periodic or almost-periodic time dependence, Rocky Mountain Journal of Mathematics 38 (2008), pp. 1147-1175. MR2436718 (2009g:47197)

[25] Y. Kametaka, On the nonlinear diffusion equation of Kolmogorov-Petrovskii-Piskunov type, Osaka J. Math. 13 (1976), pp. 11-66. MR0422875 (54:10861)

[26] C.-Y. Kao, Y. Lou, and W. Shen, Random dispersal vs. non-local dispersal, Discrete and Continuous Dynamical Systems 26 (2010), no. 2, pp. 551-596. MR2556498(2011a:35253)

[27] A. Kolmogorov, I. Petrowsky, and N. Piscunov, A study of the equation of diffusion with increase in the quantity of matter, and its application to a biological problem.Bjul. Moskovskogo Gos. Univ. 1 (1937), pp. 1-26.

[28] X. Liang and X.-Q. Zhao, Asymptotic speeds of spread and traveling waves for monotone semiflows with applications, Comm. Pure Appl. Math. 60 (2007), no. 1, pp. 1-40. MR.2270161 (2007i:37144)

[29] X. Liang and X.-Q. Zhao, Spreading speeds and traveling waves for abstract monostable evolution systems, Journal of Functional Analysis 259 (2010), no. 4, pp. 857-903. MR2652175

[30] X. Liang, Y. Yi, and X.-Q. Zhao, Spreading speeds and traveling waves for periodic evolution systems, J. Diff. Eq. 231 (2006), no. 1, pp. 57-77. MR.2287877(2008a:47110)

[31] W.-T. Li, Y.-J. Sun, and Z.-C. Wang, Entire solutions in the Fisher-KPP equation with nonlocal dispersal, Nonlinear Analysis, Real World Appl. 11 (2010), no. 4, pp. 2302-2313. MR2661901

[32] R. Lui, Biological growth and spread modeled by systems of recursions, I. Mathematical Theory, Math. Biosciences 93 (1989), pp. 269-312. MR984281 (90g:92069)

[33] G. Lv and M. Wang, Existence and stability of traveling wave fronts for nonlocal delayed reaction diffusion systems, preprint.

[34] J. Nolen, M. Rudd, and J. Xin, Existence of KPP fronts in spatially-temporally periodic advection and variational principle for propagation speeds, Dynamics of PDE 2 (2005), pp. 1-24. MR.2142338 (2006h:35129)

[35] J. Nolen and J. Xin, Existence of KPP type fronts in space-time periodic shear flows and a study of minimal speeds based on variational principle, Discrete and Continuous Dynamical Systems 13 (2005), pp. 1217-1234. MR2166666 (2006e:35175)

[36] S. Pan, W.-T. Li, and G. Lin, Existence and stability of traveling wavefronts in a nonlocal diffusion equation with delay, Nonlinear Analysis: Theory, Methods \& Applications 72 (2010), pp. 3150-3158. MR2580167 (2010m:35538)

[37] A. Pazy, Semigroups of Linear Operators and Applications to Partial Differential Equations, Springer-Verlag, New York-Berlin-Heidelberg-Tokyo, 1983. MR.710486 (85g:47061)

[38] D. H. Sattinger, On the stability of waves of nonlinear parabolic systems, Advances in Math. 22 (1976), pp. 312-355. MR0435602 (55:8561)

[39] W. Shen, Variational principle for spatial spreading speeds and generalized propagating speeds in time almost and space periodic KPP models, Trans. Amer. Math. Soc. 362 (2010), pp. 5125-5168. MR2657675

[40] W. Shen and G. T. Vickers, Spectral theory for general nonautonomous/random dispersal evolution operators, J. Differential Equations 235 (2007), pp. 262-297. MR2309574 (2008d:35091)

[41] W. Shen and A. Zhang, Spreading speeds for monostable equations with nonlocal dispersal in space periodic habitats, Journal of Differential Equations 249 (2010), pp. 747-795. MR2652153

[42] W. Shen and A. Zhang, Traveling wave solutions of monostable equations with nonlocal dispersal in space periodic habitats, submitted.

[43] K. Uchiyama, The behavior of solutions of some nonlinear diffusion equations for large time, J. Math. Kyoto Univ. 183 (1978), pp. 453-508. MR509494 (80g:35016) 
[44] H. F. Weinberger, Long-time behavior of a class of biology models, SIAM J. Math. Anal. 13 (1982), pp. 353-396. MR653463 (83f:35019)

[45] H. F.Weinberger, On spreading speeds and traveling waves for growth and migration models in a periodic habitat, J. Math. Biol. 45 (2002), pp. 511-548. MR.1943224 (2004b:92043a)

Department of Mathematics, Auburn University, Auburn, Alabama 36849

E-mail address: wenxish@auburn.edu

Department of Mathematics, Auburn University, Auburn, Alabama 36849

E-mail address: zhangai@auburn.edu 